(2) Open Access Full Text Article

REVIEW

\title{
Systematic review of randomized controlled trials of probiotics, prebiotics, and synbiotics in inflammatory bowel disease
}

This article was published in the following Dove Press journal:

Clinical and Experimental Gastroenterology

9 December 2014

Number of times this article has been viewed

\section{Yezaz A Ghouri \\ David M Richards \\ Erik F Rahimi \\ Joseph T Krill \\ Katherine A Jelinek \\ Andrew W DuPont}

The University of Texas Medical School at Houston, Houston, Texas, USA
Correspondence:Andrew W DuPont The University of Texas Medical School at Houston, 643I Fannin Street 4.234, Houston, Texas 77030, USA

$\mathrm{Tel}+\mathrm{I} 7135006687$

Fax +I 7135006699

Email Andrew.Dupont@uth.tmc.edu
Background: Probiotics are microorganisms that are ingested either in combination or as a single organism in an effort to normalize intestinal microbiota and potentially improve intestinal barrier function. Recent evidence has suggested that inflammatory bowel disease (IBD) may result from an inappropriate immunologic response to intestinal bacteria and a disruption in the balance of the gastrointestinal microbiota in genetically susceptible individuals. Prebiotics, synbiotics, and probiotics have all been studied with growing interest as adjuncts to standard therapies for IBD. In general, probiotics have been shown to be well-tolerated with few side effects, making them a potential attractive treatment option in the management of IBD.

Aim: To perform a systematic review of randomized controlled trials on the use of probiotics, prebiotics, and synbiotics in IBD.

Results: In our systematic review we found 14 studies in patients with Crohn's disease (CD), 21 studies in patients with ulcerative colitis (UC), and five studies in patients with pouchitis. These were randomized controlled trials using probiotics, prebiotics, and/or synbiotics. In patients with $\mathrm{CD}$, multiple studies comparing probiotics and placebo showed no significant difference in clinical outcomes. Adding a probiotic to conventional treatment improved the overall induction of remission rates among patients with UC. There was also a similar benefit in maintaining remission in UC. Probiotics have also shown some efficacy in the treatment of pouchitis after antibiotic-induced remission.

Conclusions: To date, there is insufficient data to recommend probiotics for use in CD. There is evidence to support the use of probiotics for induction and maintenance of remission in UC and pouchitis. Future quality studies are needed to confirm whether probiotics, prebiotics, and synbiotics have a definite role in induction or maintenance of remission in $\mathrm{CD}, \mathrm{UC}$, and pouchitis. Similar to probiotics, fecal microbiota transplantation provides an alternate modality of therapy to treat IBD by influencing the intestinal flora.

Keywords: inflammatory bowel disease, Crohn's disease, ulcerative colitis, pouchitis, probiotics, prebiotics, synbiotics

\section{Introduction}

Inflammatory bowel disease (IBD) is a chronic relapsing inflammatory disorder of the gastrointestinal tract, comprising ulcerative colitis (UC) and Crohn's disease (CD). IBD affects more than 1 million people in the United States and 2.5-3 million people in Europe. ${ }^{1,2}$ The standard treatment approach for patients with CD and UC has traditionally been with the use of anti-inflammatory agents, like mesalamine, corticosteroids, immunomodulators such as azathioprine, and biologic agents such as infliximab. Although the exact pathophysiology of IBD is unknown, the current 
paradigm is multifactorial and involves an abnormal intestinal immune response to the gut microbiota in a genetically susceptible host. ${ }^{3}$

Interest in microbiota-based therapy has grown markedly, due in large part to an improved safety profile with fewer side effects when compared to traditional therapy. It is important to note, however, that there are only a limited number of large, well-designed prospective trials that could evaluate for potential rare side effects in IBD patients, many of whom are immunocompromised. Collectively, the gut microbiota contains a total of $10^{13}$ to $10^{14}$ microorganisms, with the majority (10 ${ }^{12}$ microorganisms) hosted within the colon. ${ }^{4}$ Firmicutes and Bacteroidetes are the main bacterial phyla in the gut, comprising about $90 \%$ of the microbiota, followed to a lesser proportion by Actinobacteria, Proteobacteria, Verrucomicrobia, Fusobacteria, and Cyanobacteria. ${ }^{5}$ Furthermore, mucosaassociated bacterial communities in the colon are significantly different in composition from those in feces. ${ }^{6}$

Studies in human and mouse models over the past several years have investigated the relationship between dysbiosis of the gut microbiota and intestinal diseases. Mouse models have further advanced our understanding of the pathophysiology of IBD, showing that bacteria play a fundamental role in the initiation and development of chronic intestinal disease. A pivotal study showed that germfree interleukin (IL)-10-deficient mice did not develop colitis compared to IL-10 deficient mice with normal enteric bacteria. ${ }^{7}$ Other studies have shown that germfree mice given dextran sodium sulfate, which induces colitis, developed more severe disease than did non-germfree mice, suggesting a protective effect of the microbiota. ${ }^{8}$ In human model analysis using $16 \mathrm{~S}$ ribosomal (r)RNA sequencing, the microbiota of IBD patients differed from that of healthy individuals. In IBD, the diversity and bacterial load of Bacteroidetes and Firmicutes are decreased, especially in regions of active inflammation. These groups of bacteria are particularly important as they produce short-chain fatty-acid metabolites. The short-chain fatty-acid substrates have potent anti-inflammatory properties, are important sources of energy for colonic epithelial cells, and may enhance epithelial barrier integrity. Finally, microbiota changes in IBD have a higher proportion of Actinobacteria and Enterobacteria, phyla which contain many of the known gut pathogens. ${ }^{9}$

Because of this association between the intestinal microflora and IBD, multiple studies have investigated the use of probiotics, prebiotics, and synbiotics to modify the gut microbiota in order to replace or augment conventional IBD therapies. Probiotics have been defined as live microorganisms that confer a health benefit to the host when ingested. Prebiotics are nondigestible substances acting as a nutritive substrate to stimulate the growth and metabolism of protective endogenous enteric bacteria. Synbiotics are a combination of both probiotics and prebiotics. ${ }^{10}$ Probiotics are believed to contribute to the normal functioning of the intestinal commensal microbiota. They also displace pathogenic microbes, improve host mucosal barrier function, and modulate the innate host immune function. ${ }^{11}$ Finally, recent data suggest that a new approach to altering the gut microbiota by fecal microbiota transplantation has gained popularity in the treatment of Clostridium difficile infection and may be a promising option in treating IBD, as well. ${ }^{12}$ For that reason it also will be discussed in this review.

Multiple studies have been done recently on the use of probiotic, prebiotic, and synbiotic agents as an adjuvant therapy in the treatment of CD and UC. Treatment approaches for these conditions can be divided into treatment during an acute flare (induction therapy) and treatment for long-term control of symptoms (maintenance therapy). Pouchitis is a clinical diagnosis for nonspecific, idiopathic inflammation of the ileal reservoir created by an ileal pouch-anal anastomosis, which is a commonly employed procedure following total proctocolectomy for patients with UC. ${ }^{13}$ Previous studies have shown clinical benefit of some probiotics in pouchitis and UC, but less clearly in CD. ${ }^{14-17}$ The aim of the present article is to provide an overview of randomized controlled trials (RCTs) in human studies of CD, UC, and pouchitis and to draw practical clinical conclusions about the efficacy of probiotics, prebiotics, and synbiotics in treating patients with these conditions.

\section{Methods}

We performed a literature search in Medline, PubMed, Cochrane database, Science Direct, Ovid, and Scopus. The results were limited to full-text articles published in English. The search terms used were "inflammatory bowel disease", "IBD”, "inflammation", "ulcerative colitis", "Crohn's disease", and "pouchitis". These terms were cross-referenced with the terms "probiotic", "prebiotic", and "synbiotic" in all possible combinations. Only RCTs performed among human subjects were selected for this review. Studies both in pediatric and adult populations were included. The initial search yielded 4,440 results: the search terms "inflammation" or "inflammatory bowel disease" and "prebiotic", "probiotic", or "synbiotic" yielded 3,188 results; the remaining 1,252 results were obtained by searching the individual types of inflammatory bowel disease with "probiotic", "prebiotic", or "synbiotic". Several of these results provided redundant articles. 
The authors reviewed the titles and/or abstracts to exclude studies that were not RCTs performed with IBD patients using probiotics, prebiotics, or synbiotics. We excluded any animal studies or human cell or tissue culture studies. Those trials that did not define clear randomization of the control and the study group were excluded. After applying the exclusion criteria, we selected 82 studies for further review. Fulltext versions of these articles were reviewed in detail by the authors. Finally, 40 RCTs were selected for inclusion in our systematic review, including 14 CD studies, 21 UC studies, and five pouchitis studies. The excluded articles consisted of nine CD studies, $26 \mathrm{UC}$ studies, and seven pouchitis studies. A description of the article selection process is shown in Figure 1.

\section{Results \\ Crohn's disease}

In our literature search we found 14 RCTs that aimed to determine the outcome following probiotic, prebiotic, or synbiotic treatment for the management of $\mathrm{CD}^{18-31}$ (described in Table 1). One study was performed among $\mathrm{CD}$ and UC patients. ${ }^{30}$ Ten studies included only probiotics in the intervention group. ${ }^{19-25,27,31}$ Of these, five used Lactobacillus. ${ }^{21-25}$ The species Lactobacillus GG (LGG) was used in three trials and Lactobacillus johnsonii was used in two trials. ${ }^{21-23}$ The primary end point in three studies was endoscopic recurrence rate and in two studies was clinical relapse rate. The Crohn's Disease Activity Index for adults

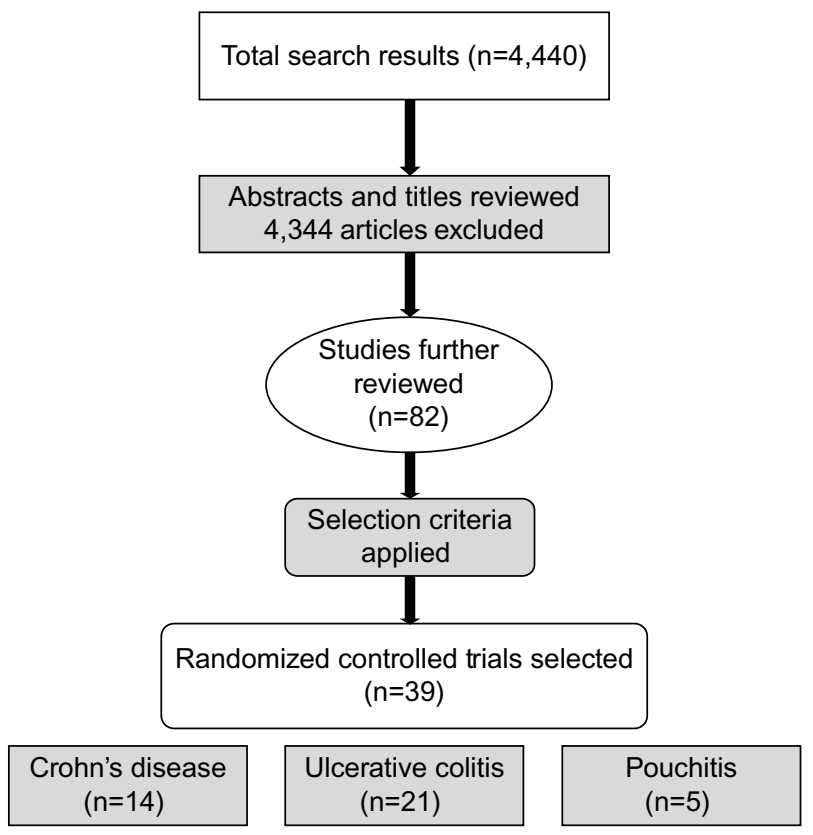

Figure I Flowchart showing randomized controlled study selection criteria for inclusion in the systematic review.
(CDAI, which measures the clinical severity of CD and helps in determining if there is active disease, relapse and severity of relapse $)^{32}$ was used in eight studies conducted in the adult population. ${ }^{20,22,24,25,27-29,31}$ A study by Bousvaros et $\mathrm{al}^{23}$ performed in a pediatric population used the Pediatric Crohn's Disease Activity Index score ${ }^{33}$ to evaluate the severity of clinical symptoms; this study was stopped prematurely at 42 months because an interim data analysis showed no efficacy with use of the probiotics and because enrolling the pediatric population was becoming more challenging for the long duration of the study. In essence, none of the Lactobacillus-containing probiotics used in the CD study populations showed any meaningful effect on endoscopic remission or on the CDAI. Among the other five probioticonly trials, Saccharomyces boulardii was used in four studies. ${ }^{18,20,27,31}$ One study by Malchow ${ }^{19}$ conducted in 1997 among CD patients used Escherichia coli strain Nissle 1917 This was a double-blinded placebo-controlled pilot study conducted over 1 year in 28 patients who were in remission from $\mathrm{CD}$. The remission rates among the probiotic and placebo groups were not statistically different. The study also showed that E. coli strain Nissle 1917 produced no clinical harm among the study population.

Plein and Hotz ${ }^{18}$ performed a pilot study in 1997 using S. boulardii. This was a double-blinded placebo-controlled trial conducted among 17 patients with CD. The study showed clinical benefit in decreasing the frequency of stools and improving CDAI scores. ${ }^{32}$ In 2000, a study published by Guslandi et $\mathrm{al}^{20}$ showed clinical benefit with use of $S$. boulardii as determined by a decrease in the rate of clinical relapse, which was measured by the CDAI; however, the study contained only 16 patients in each of the control and intervention groups. In 2013, Bourreille et $\mathrm{al}^{31}$ published a study comparing the use of $S$. boulardii in 84 patients with 81 controls. This larger trial did not show any clinical benefit in relapse rate as determined by the CDAI score at the end of 40 weeks of follow-up. The study was well designed and included a total of 165 individuals. The final study from Garcia Vilela's group $^{27}$ in 2008 examined 14 and 17 CD patients in remission (defined by CDAI) who were treated with $S$. boulardii or placebo, respectively. They measured intestinal permeability with a lactulose:mannitol ratio, which has been shown to be associated with increased disease activity. While this study showed a reduction in the lactulose:mannitol ratio in the probiotic group, the data were difficult to interpret or apply given the wide variability of intestinal permeability in $\mathrm{CD}$ patients and lack of correlation with CDAI or endoscopic remission. These three studies described above showed benefit in using 


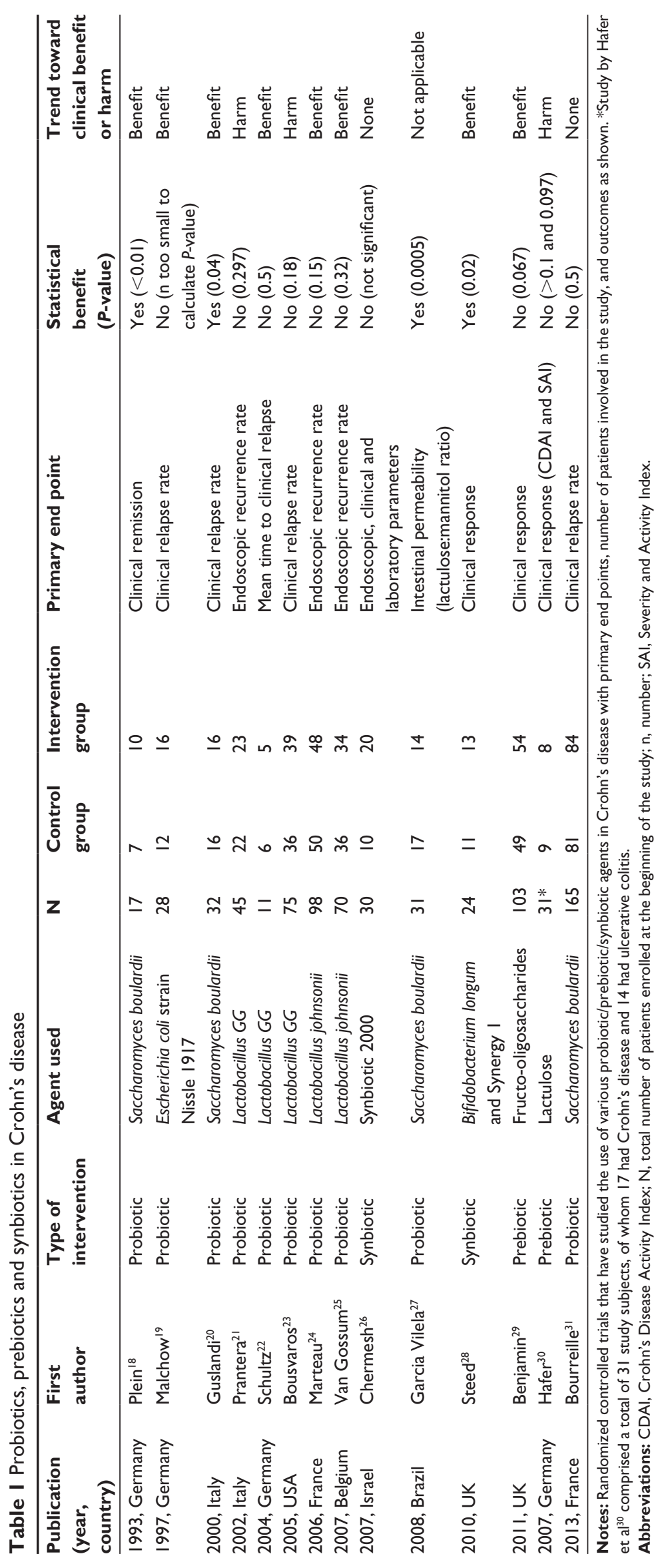


S. boulardii, but their major limitation was the small size of the study populations.

Studies using prebiotics and synbiotics in $\mathrm{CD}$ are further described. Two studies were found using only prebiotics for therapy in $\mathrm{CD}^{29,30}$ In 2011, Benjamin et a ${ }^{29}$ performed a study with 103 subjects ( 49 in the control group and 54 in the intervention group) using fructo-oligosaccharides. Clinical response was measured using CDAI score; however, there was no clinical benefit at the end of 4 weeks of therapy. Another trial using lactulose as a prebiotic was performed in 2007 by Hafer's group among patients with $\mathrm{UC}$ and $\mathrm{CD} .{ }^{30}$ There was no clinical benefit in treatment among the $\mathrm{CD}$ patients but improvement in quality of life was seen in those with UC. Hafer et al ${ }^{30}$ used the CDAI and Severity and Activity Index ${ }^{34}$ scoring systems to determine clinical severity of the disease. Both studies showed no improvement in the scores in the prebiotic group; in fact, placebo groups showed a better response.

Two studies evaluated the use of a synbiotic preparation among patients with $\mathrm{CD}^{26,28}$ One study, published in 2010 by Steed et al, ${ }^{28}$ consisted of eleven patients in the placebo group in comparison to 14 in the synbiotic group. The synbiotic preparation consisted of Bifidobacterium longum and Synergy 1, a preferential growth substrate consisting of an inulin/oligofructose mix. At the end of 3 months and 6 months of therapy, there was significant improvement in CDAI scores in the synbiotic group in comparison to the placebo group. A major limitation of the study was that the baseline CDAI scores were higher in the placebo group. A second synbiotic study conducted by Chermesh et $\mathrm{al}^{26}$ used Synbiotic 2000, a combination of four probiotic and four prebiotic agents. It consisted of four lactic acid-producing bacteria: Pediacoccus pentoseceus, Lactobacillus affinolactis, Lactobacillus paracasei susp paracasei 19 and Lactobacillus plantarum 2362. It also consisted of four fermentable fibers: $\beta$-glucans, inulin, pectin, and resistant starch. The study included ten patients in the placebo group and 20 in the intervention group. Clinical, laboratory, and endoscopic parameters were used to compare the two groups for 24 months. At completion of the study, there was no statistically significant benefit among the intervention group in terms of improvement in clinical symptoms (CDAI score), endoscopic features (Rutgeerts' scoring system) ${ }^{35}$ or laboratory measurements. Given the findings above, there are no clinical benefits found with prebiotics and synbiotics in patients with $\mathrm{CD}$.

\section{Ulcerative colitis}

Probiotics, prebiotics, and synbiotics have all been studied as therapeutic adjuncts in the management of ulcerative colitis.
A total of 21 RCT studies were identified that evaluated the use of various probiotics, prebiotics, and/or synbiotics used as induction therapy, maintenance of remission, and/ or combination of both in patients with UC.

\section{Ulcerative colitis - induction}

Our literature search found nine studies using various types of probiotics, prebiotics, and synbiotics for induction therapy in $\mathrm{UC}^{30,36-43}$ (described in Table 2). The majority of these studies enrolled UC patients with mild to moderate disease activity, with the largest trials using VSL\#3. VSL\#3 is a multibacterial culture for oral use, consisting of the following species: Bifidobacterium breve, B. longum, Bifidobacterium infantis, Lactobacillus acidophilus, L. plantarum, L. paracasei, Lactobacillus bulgaricus, and Streptococcus thermophilus. ${ }^{44}$ Tursi et a ${ }^{36}$ in 2004 evaluated 30 patients receiving VSL $\# 3$ at a dose of 900 billion bacteria daily with low-dose balsalazide, $30 \mathrm{UC}$ patients with medium-dose balsalazide alone, and another $30 \mathrm{UC}$ patients with mesalamine alone. The rate of clinical remission was comparable between low-dose balsalazide with VSL\#3 and medium-dose balsalazide, but statistical analysis showed that a daily dose of VSL\#3 with balsalazide was significantly superior to mesalamine in achieving symptomatic remission. This dose of VSL\#3 was lower than the dose used in the largest of the UC induction of remission studies done by Sood et $\mathrm{al}^{39}$ in 2009 and Tursi et $\mathrm{al}^{40}$ in 2010. These two studies used VSL $\# 3$ at a dose of 3600 billion bacteria daily. These two studies enrolled 147 and 144 patients, respectively, with mild to moderate UC and concomitant use of aminosalicylates or thiopurines. Both studies showed a decrease in the Ulcerative Colitis Disease Activity Index ${ }^{45}$ by $50 \%$ or more in the treatment arm. At 12 weeks, Sood et a ${ }^{39}$ observed that $42.9 \%$ of the VSL\#3 treated patients had achieved remission compared with $15.7 \%$ of patients with placebo $(P<0.001)$. Tursi et a $1^{40}$ observed that $47.7 \%$ of patients treated with VSL\#3 were in remission compared to $32.4 \%$ in the placebo group; however this did not reach statistical significance. Limitations of this study include a high placebo response rate and relatively short duration of study.

On a cellular level, VSL\#3 affected dendritic cells, which shape bacterial recognition and T-cell responses, in patients with acute UC in a study by $\mathrm{Ng}$ et al. ${ }^{41}$ There was an increase in anti-inflammatory IL-10 production and decrease in proinflammatory IL- $12 \mathrm{p} 40$ production by colonic dendritic cells following VSL\#3 treatment. These data suggest an association between a change toward beneficial dendritic cell cytokine profiles and clinical efficacy. 


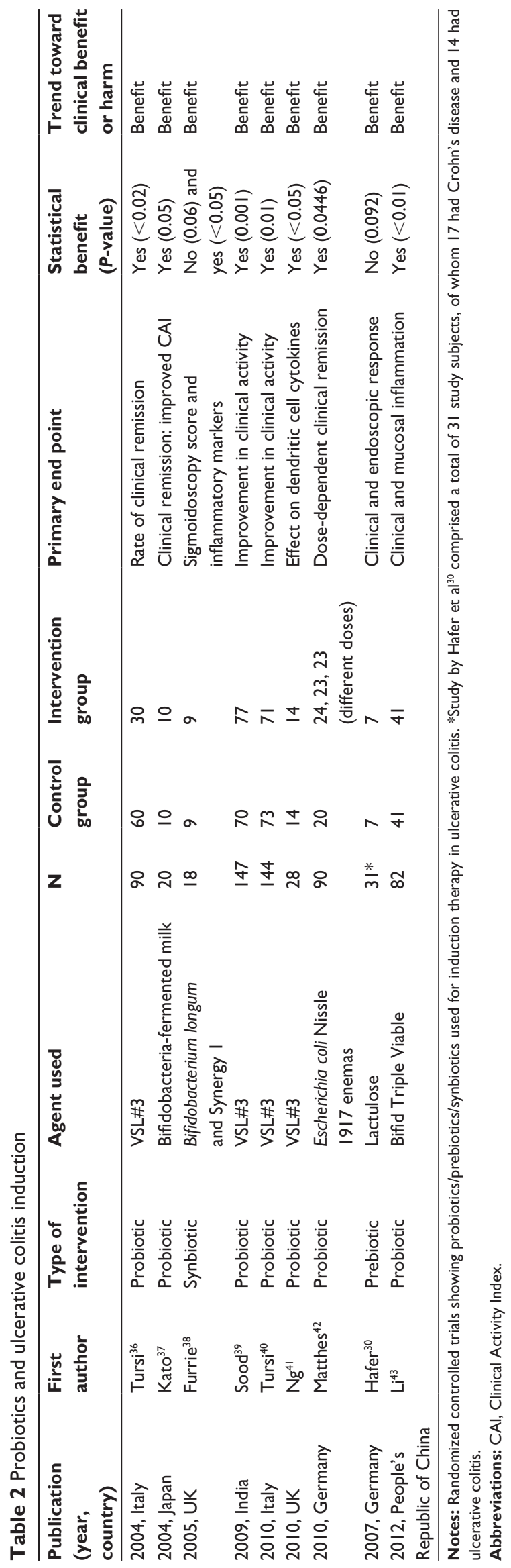

Similar increased IL-10 production was seen in patients treated with steroids.

Three other trials evaluated other probiotic regimens and one trial evaluated a synbiotic regimen in remission of UC. Bifidobacteria-fermented milk (BFM), which contains Bifidobactrium strains and L. acidophilus, was studied by Kato et $\mathrm{a}^{37}$ in a small study of 20 patients. They achieved their primary end point of a decrease in the Clinical Activity Index (CAI) score ${ }^{46}$ of at least 3 points by 12 weeks. The BFM-treated group's CAI score was significantly lower than the placebo score at 12 weeks $(P<0.05)$ along with improved Endoscopic Activity Index ${ }^{47}$ and histological scores. Li et al ${ }^{43}$ evaluated the use of Bifid Triple Viable, which is a combination of Bacillus acidophilus, Bifidobacterium bifidum, and fecal streptococci, combined with 5-aminosalicylic acid compared to patients taking 5-aminosalicylic acid alone. Clinical symptoms based on the Mayo Score ${ }^{48}$ and colonic mucosal inflammation improved more in the treatment group. Matthes et $\mathrm{al}^{42}$ evaluated the efficacy of $E$. coli strain Nissle 1917 in patients with proctitis and proctosigmoiditis. This was the only study that evaluated delivery of a probiotic via an enema for the treatment of acute UC. Their study examined several different doses of the probiotic, which resulted in small subgroups. Per protocol analysis indicated a statistically significant difference in dose-dependent efficacy $(P=0.0446)$; however, they failed to show statistical significance in the intent-to-treat analysis.

A small study done by Furrie et a ${ }^{38}$ used a novel synbiotic consisting of $B$. longum and a combination of a prebiotic (Synergy 1) for the probiotic strain for 4 weeks. Much of their focus was on the effects of the treatment with respect to inflammatory markers and the presence of the bacteria in mucosal biopsies. There was a statistically significant reduction in the inflammatory markers among the synbiotic group when compared to the placebo group. Clinically they observed a trend toward improved sigmoidoscopy scores (without statistical significance) and no significant difference in CAI scores. However the data were limited by both a small study population $(\mathrm{n}=18)$ and a high dropout rate. As mentioned previously, Hafer et al, ${ }^{30}$ published a study in 2007 which showed no clinical or endoscopic benefit in the use of lactulose as a prebiotic among $17 \mathrm{CD}$ and $14 \mathrm{UC}$ patients. However, UC patients showed an improvement in the quality of life. Use of the prebiotic in $\mathrm{CD}$ and UC showed no benefit in terms of clinical activity index, endoscopic score, or immunohistochemical parameters; however, UC patients showed an improvement in the quality of life. The study was limited by the size of the population and lack of placebo use in 
the control group, meaning that we cannot rule out a placebo effect in the treatment group.

\section{Ulcerative colitis - maintenance}

Nine studies have been published examining the use of probiotics in the maintenance of UC, and three studies evaluated both combined induction and maintenance of remission in UC. These 12 studies ${ }^{49-60}$ are described in Table 3.

A study by Kruis et $\mathrm{al}^{49}$ in 1997 using E. coli Nissle 1917 in comparison with mesalamine in a population of 103 patients showed no benefit in the probiotic group in comparison with the mesalamine group. Another study by Kruis et $\mathrm{al}^{52}$ in 2004 randomized 327 patients to receive either E. coli Nissle $1917(\mathrm{n}=162)$ or mesalamine $(\mathrm{n}=165)$. The E. coli group had a relapse rate of $56.4 \%$ compared to $33.9 \%$ in the placebo group over a 12 month period $(P=0.003)$. The CAI trended towards a greater increase in the E. coli group; however, there were no statistically significant differences in quality of life score, endoscopy scores, and histology scores. In 2011, Wildt et $\mathrm{al}^{57}$ enrolled 32 patients with left-sided $\mathrm{UC}$ in remission to take either placebo $(\mathrm{n}=12)$ or a probiotic consisting of L. acidophilus La-5 and Bifidobacterium animalis subspecies lactis BB-12. Although no statistically significant benefit was seen in the primary end point of maintaining remission, there was a trend toward increased remission in the probiotic group. In 2004, Cui et al ${ }^{53}$ studied 30 patients with UC initially treated with sulfasalazine and glucocorticoids and then randomly administered either Bifid Triple Viable capsule or an identical placebo (starch). Fecal analysis and histological analysis were performed after 8 weeks or in cases of relapse. The study showed that high concentration of luminal gram-positive bacteria were seen in the probiotics group $(n=15)$, which could prolong the time to relapse of ulcerative colitis. Also, proinflammatory cytokines were inhibited by probiotics, and messengerRNA expression of anti-inflammatory cytokine IL-10 was elevated by the effect of probiotics. In 2010, Hegazy and El-Bedewy ${ }^{58}$ used a probiotic preparation containing Lactobacillus delbruekii and Lactobacillus fermentum for 8 weeks in newly diagnosed UC patients $(n=15)$ who were being treated with sulfasalazine compared to those taking sulfasalazine and placebo. Histological improvement of inflammation was significantly improved in the probiotic group compared to the sulfasalazine group and was associated with decreased fecal calprotectin and mucosal inflammatory cytokines.

There were two open-label prospective RCTs, one evaluating the use of probiotics and the other evaluating the use of synbiotics for maintenance therapy in UC. Ishikawa et $\mathrm{al}^{51}$ in
2003 enlisted 21 patients in an open-label RCT using BFM (B. breve, B. bifidum, and L. acidophilus) combined with standard therapy in eleven patients. There were significantly fewer episodes of symptomatic exacerbations in the probiotic group observed over a period of one year when compared to standard therapy ( $27 \%$ versus $90 \%, P=0.0075)$. In 2006 , Zocco et $\mathrm{al}^{54}$ studied a sample size of 187 patients in an openlabel RCT using LGG as the probiotic with three treatment groups: LGG alone, LGG combined with mesalamine, and mesalamine alone. The primary end point was the number of relapses over 12 months. There was no significant difference in the relapse rates among the three groups, and the authors concluded that they established equivalent efficacy. Fujimori et al, ${ }^{55}$ in 2009 , also conducted a large $(n=120)$ prospective open-label RCT using a synbiotic in patients with UC who were either in remission or had mildly active disease. The patients were randomly assigned into one of the three groups: probiotic (B. Longum) group, prebiotic (psyllium) group and synbiotic (a combination of the aforementioned prebiotic and probiotic) group. We arbitrarily have labeled these three groups as group a, b, and c in Table 3 . The primary end point was scores on the Inflammatory Bowel Disease Questionnaire, ${ }^{61}$ which assesses health-related quality of life in IBD, at 4 weeks. Results showed a statistically significant improvement in scores for the synbiotic group at the end of the study. This improvement was not seen in the probiotic or prebiotic groups. The limitations of this study included a high dropout rate, no endoscopic or histological examinations, and short duration. In 2012, Oliva et $\mathrm{al}^{60}$ performed a double-blinded RCT among a pediatric population with UC in remission. Fifteen controls received placebo and mesalamine and 16 patients received a probiotic, Lactobacillus reuteri ATCC 55730 with mesalamine. The results of the study showed improvement in clinical and endoscopic findings (Mayo score) and decrease in severity of inflammation/inflammatory markers in the probiotic group.

\section{Ulcerative colitis - induction and maintenance}

The persistent effect of both induction and maintenance of remission in UC was evaluated in three double-blind RCTs. In 1999, Rembacken et al ${ }^{50}$ studied 116 patients with active UC over a period of 12 months. Patients received pretreatment with oral gentamicin followed by a nonpathogenic strain of E.coli Nissle 1917 serotype O6:K5:H1 and were compared to patients receiving pretreatment with gentamicin and standard treatment with mesalamine with respect to induction of remission and prevention of relapse. Thirty-nine of 57 patients $(68 \%)$ in the $E$. coli group achieved remission compared to 


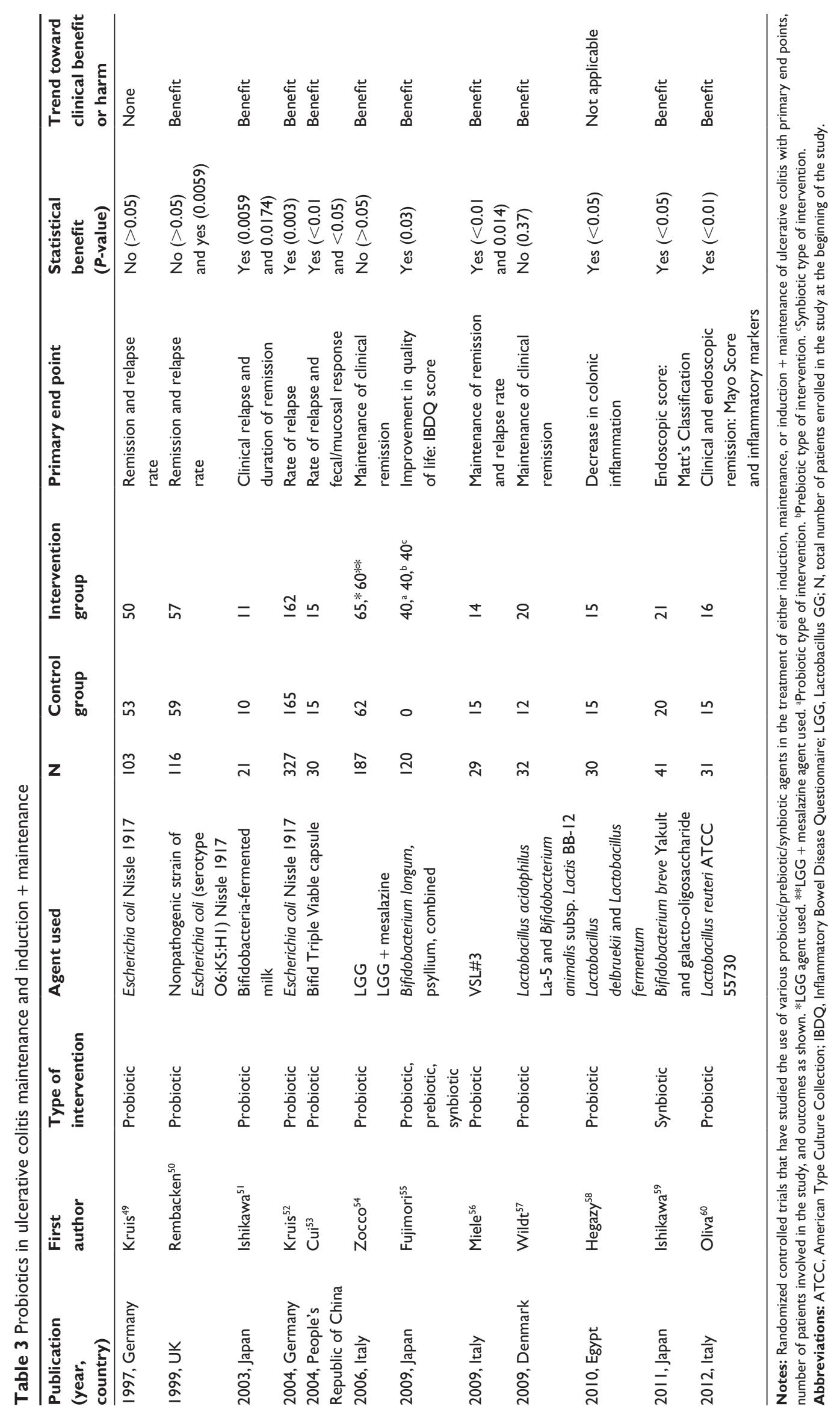


$44(75 \%)$ in the mesalamine group, which was not statistically significant $(P>0.05)$. Thirty-two patients $(73 \%)$ in the mesalamine group relapsed within 1 year compared with 26 (67\%) in the E. coli group, which was borderline statistically significant $(P=0.059)$. The study showed that $E$. coli Nissle 1917 is equivalent to mesalamine in maintaining remission. A smaller study of 29 patients in 2009 by Miele et al ${ }^{56}$ evaluated the use of VSL\#3 in children with UC for both the induction and maintenance of remission over a 1-year period in conjunction with standard therapy. They observed higher remission rates in the group treated with VSL\#3 compared to placebo ( $92.8 \%$ versus $36.4 \%, P<0.001$ ), although the mean duration for achieving induction with standard steroid therapy was not significantly different between the two groups. In 2011, Ishikawa et al ${ }^{59}$ studied 41 patients, of whom 21 took a synbiotic preparation containing a $B$. breve strain (Yakult) as its probiotic component and galacto-oligosaccharide as the prebiotic component. The patients were followed for 1 year and evaluated for endoscopic improvement. The study reported improvement of endoscopic grading (Matt's Classification $)^{62}$ in the synbiotic group when compared to the standard therapy group.

\section{Pouchitis}

Data regarding the effectiveness of probiotics for induction and maintenance therapy in pouchitis is very limited. There were no RCTs evaluating the use of prebiotics and synbiotics for management of pouchitis. A total of five RCTs using probiotics in pouchitis were found ${ }^{63-67}$ (listed in Table 4). In all the studies, disease activity was measured by the Pouchitis Disease Activity Index (PDAI), which is an 18-point scale consisting of three categories: symptoms, endoscopic findings, and histological findings. ${ }^{68}$ Four of the trials used VSL\#3 as the probiotic agent ${ }^{63,65-67}$ and one trial used only LGG. ${ }^{64}$ Four of the five RCTs found probiotics to be effective in the maintenance of remission in pouchitis. ${ }^{63,65-67}$ One trial found that there was no such effect. ${ }^{64}$ The doses of culture selected for use were not uniform between these studies.

In 2000, Gionchetti et al ${ }^{63}$ published a double-blinded, placebo-controlled trial that used probiotic VSL\#3 as a maintenance treatment in patients with chronic relapsing pouchitis, defined as $>3$ relapses per year. This study involved 40 patients who were in clinical and endoscopic remission after 1 month of antibiotic treatment with ciprofloxacin and rifaximin. Patients were randomized to receive $6 \mathrm{~g}$ daily of VSL\#3 (1,800 billion bacteria/daily dose) or an identical placebo for 9 months. The patients were assessed clinically every month and endoscopically and histologically every

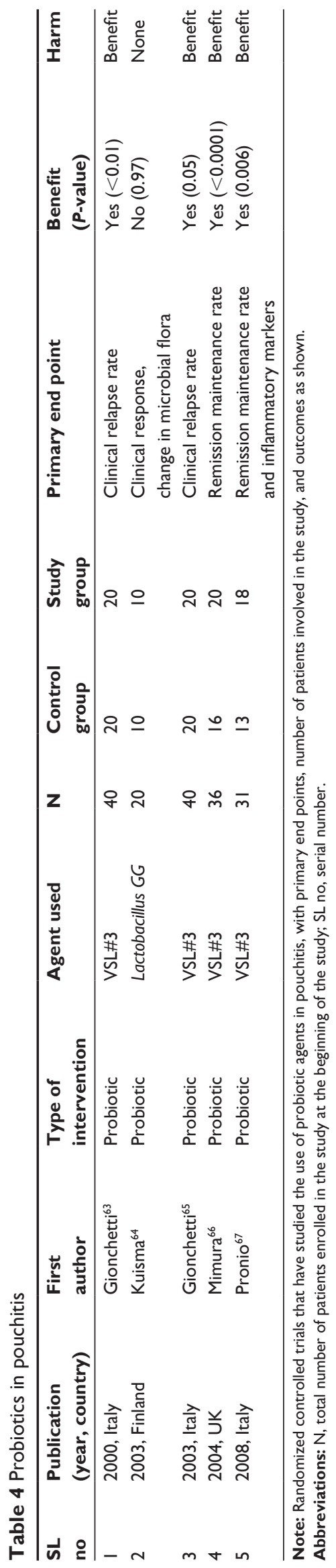


2 months or in the event of a relapse. Relapse was defined as an increase of $>2$ points in the clinical category of PDAI and was confirmed by endoscopic and histological findings. At the end of 9 months, $15 \%$ of the treatment group experienced a relapse while $100 \%$ in the placebo group experienced a relapse $(P<0.01)$. A subsequent double-blinded RCT in 2003 by Gionchetti et a ${ }^{65}$ evaluated 40 patients for 1 year who were randomized to VSL\#3 or placebo for disease maintenance in pouchitis. These patients underwent clinical, endoscopic, and histological assessment at 1 month, 3 months, 6 months, 9 months, and 12 months. Development of pouchitis occurred in $10 \%$ of those in the treatment group compared to $40 \%$ of those treated with placebo $(P<0.05)$. Additionally, the treatment group demonstrated significant improvement in quality of life, lower stool frequency, and increased concentrations of "protective bacteria".

Pronio et $\mathrm{al}^{67}$ conducted an open-labeled RCT involving 31 UC patients who had undergone protocolectomy with ileal pouch-anal anastomosis who had no signs or symptoms of pouchitis. These patients were randomized to receive either VSL\#3 daily (450 billion bacteria/packet) or no treatment. They were evaluated clinically and endoscopically at baseline and after 3 months, 6 months, and 12 months. Patients with a total PDAI score $>7$ were defined as having acute pouchitis. Patients treated with VSL\#3 showed a small but statistically significant reduction in PDAI score at 3 months $(P=0.014)$ and maintained these scores at 6 months and 12 months, compared to no change in PDAI score in the placebo group. In addition, the study found that VSL\#3 administration led to an expansion of mucosal regulatory T-cells that was speculated to have a role in controlling mucosal inflammation.

Mimura et al $^{66}$ conducted a double-blinded RCT involving 36 patients who had experienced pouchitis at least twice in 1 year or who required continuous antibiotics. Initially, remission was induced with oral antibiotic therapy consisting of metronidazole and ciprofloxacin for 4 weeks. The patients were randomized to receive either VSL\#3 daily (300 billion bacteria/g) or placebo for 1 year or until relapse. Their PDAI score was evaluated before randomization, at 2 months, and at 12 months or until experiencing relapse. Remission was maintained in $85 \%$ of the treatment group compared to only $1 \%$ in the control group $(P<0.0001)$. Kuisma et $\mathrm{al}^{64}$ conducted a double-blinded RCT that used LGG. In this study, 20 patients with a history of pouchitis were randomized to receive either two capsules of LGG twice daily $(n=10$; $[0.5-1.0] \times 10^{10}$ colony-forming units/capsule) or a placebo $(n=10)$ for 3 months. The effect was measured by fecal samples and mucosal biopsies taken for bacteriological analysis and fecal bile acid composition before and after treatment. LGG was associated with altered pouch flora; however, no significant difference in PDAI scores was seen between the treatment and control group. There was no difference in clinical, endoscopic, or histological findings in the patients who were given the probiotic.

\section{Discussion \\ Crohn's disease}

There have been several studies evaluating the effect of probiotics in CD. Different probiotics have shown variable results with respect to clinical response when compared to a placebo or standard treatment. In a meta-analysis by Rahimi et al, ${ }^{69}$ eight placebo-controlled RCTs were selected, of which six using Lactobacillus showed no superior benefit in maintaining remission and preventing clinical or endoscopic relapses. Interestingly, two studies that showed some clinical benefit did not use Lactobacillus; one used Saccharomyces and the other used E. coli. This clearly suggests that use of Lactobacillus as a single probiotic agent is of no benefit in CD. In our systematic review of the literature we have found a similar effect. After surgical resection, probiotics have been used to maintain remission among $\mathrm{CD}$ patients. A meta-analysis of seven studies by Doherty et $\mathrm{al}^{70}$ in 2010 showed similar results as described above. Recently, Shen et $\mathrm{al}^{71}$ published a systematic review with meta-analysis of RCTs that evaluated the effect of probiotics in IBD patients. They selected a total of 23 trials and performed a meta-analysis that showed therapeutic benefit with the use of probiotics among UC and pouchitis patients, but no such effect was noted in CD.

In our review there were a total of 14 RCTs that studied the use of probiotics, prebiotics, and/or synbiotics in CD. ${ }^{18-31}$ Of these, four showed a benefit in outcome with use of intervention. ${ }^{18,20,27,28}$ Three of the four used a probiotic ${ }^{18,20,27}$ and one used a synbiotic in the study arm. ${ }^{28}$ Three studies using S. boulardii showed a benefit with treatment; however these were limited by small study populations ranging from 17 to 31 patients per study (average 26.6). ${ }^{18,20,27}$ A 2013 study using $S$. boulardii was performed in 165 patients by Bourreille et al. ${ }^{31}$ This was a well-designed RCT that showed no statistically significant difference between use of placebo and the probiotic. Of the 14 RCTs, two additional studies showed no trend toward clinical benefit or harm. ${ }^{26,31}$ There is a need for subsequent large, well-designed RCTs to evaluate the efficacy of other probiotic agents in CD.

The majority of the listed studies had significant limitations, including small study populations, high dropout rates, and lack of dose-response analyses. There was also a lack of 
consistency with the probiotic agent, dose, and concomitant use of other medications that may affect outcomes, including antidiarrheals, aminosalicylates, immunomodulators, and corticosteroids. These studies also did not correlate CDAI scores with endoscopic remission. Therefore, most of these studies provide inconclusive evidence of a beneficial role of probiotics in the induction or maintenance of remission in CD. Well-designed RCTs are needed to determine if there is any benefit with the use of probiotics, prebiotics, or synbiotics in the treatment of CD.

\section{Ulcerative colitis}

We reviewed 21 RCTs evaluating the use of various probiotics, prebiotics, and synbiotics in the management of UC. Overall, a myriad of different agents with varying doses makes drawing a conclusion regarding efficacy in achieving and/or maintaining remission in UC difficult. The limited size of the populations studied, differences in methodology, and duration of therapy limit the quality of some of the studies. The majority of studies (19/21) evaluating different agents for either induction and/or remission in mild to moderate UC showed at least a trend toward clinical benefit, ${ }^{30,36-43,50-57,59,60}$ with 16 of the 19 studies showing statistically significant benefit. $^{36-43,50-53,55,56,59,60}$ A Cochrane review in 2007 evaluated the use of probiotics for the induction of remission in UC. ${ }^{72}$ Four studies met criteria and the review concluded that probiotics combined with conventional therapy do not improve overall remission rates; however they may provide modest benefits in terms of reduction of disease activity in mild to moderate UC. The two largest studies in our review conducted after 2008 by Sood et $\mathrm{al}^{13}$ and Tursi et $\mathrm{al}^{40}$ support the use of VSL\#3 in the induction of remission. The caveat is that VSL\#3 was used as an adjunct to standard therapy, with greater than $90 \%$ of patients in the Tursi et $\mathrm{al}^{40}$ trial on mesalamine and none of the patients receiving VSL\#3 alone. In the Sood et al ${ }^{39}$ trial, $96 \%$ of patients were on mesalamine, whether alone or in combination with other UC medications, with only $2.6 \%$ of patients receiving VSL\#3 monotherapy. Currently there seems to be insufficient data to recommend for or against the use of other probiotics for the induction of remission in UC.

A Cochrane review by Naidoo et $\mathrm{al}^{73}$ in 2011 evaluated the efficacy and safety of probiotics for the maintenance of remission in UC. Four studies met inclusion criteria and tested the effect of probiotics among 587 patients with UC in remission. The studies were either small or had methodological flaws. There was no clear benefit for probiotic treatment compared to either placebo or mesalamine. While
Kruis et $\mathrm{al}^{52}$ and Zocco et $\mathrm{al}^{54}$ were among the four studies included in the Cochrane review, they do provide the best evidence that there is a role for probiotics in maintenance of remission in UC. E. Coli Nissle 1917 and LGG, which were the two probiotics used in these studies, have been studied in other types of IBD, as noted elsewhere in this review, and according to these studies they appear safe. The findings of these studies need to be replicated in larger double-blinded RCTs. At this stage it is difficult to argue superiority of a specific probiotic. Both of these authors acknowledge the efficacy of various probiotic regimens. Zocco et al ${ }^{54}$ state that the same probiotic should not be assumed to be "equally suitable for all individuals and the heterogeneity of clinical disorders such as UC suggests that strain-specific properties may be required for different patient categories".

There are limited studies evaluating the use of prebiotics in UC. Our review included one study by Hafer's group ${ }^{30}$ that showed no statistical benefit with use of prebiotic lactulose but did showed a trend toward clinical benefit. Fujimori's group ${ }^{55}$ conducted a head-to-head trial comparing the efficacy of probiotic, prebiotic, and synbiotic in UC. This study showed a superior benefit with the use of synbiotic compared to probiotic or prebiotic alone. In conclusion, larger well-designed RCTs are needed to further determine whether probiotics, type of probiotics, and/or synbiotics are of clear benefit for both the induction and/or the maintenance of remission in $\mathrm{UC}$.

\section{Pouchitis}

Since pouchitis has a similar pathogenic mechanism as UC, we expected a similar clinic benefit as seen in UC. Our review included five RCTs that evaluated the effect of probiotics in pouchitis (Table 4). In 2008, Elahi et al ${ }^{74}$ published a systematic review and a meta-analysis of five placebocontrolled RCTs that showed clinical benefit, determined by PDAI, with the use of probiotics VSL\#3 and LGG. Of the five trials selected in our review, four showed benefit in clinical outcome ${ }^{63,65-67}$ and one showed no outcome benefit in terms of clinical and endoscopic findings. ${ }^{64}$ This study used LGG culture as the probiotic agent. Among the four trials that showed benefit, the observed treatment effects were statistically significant, with some trials demonstrating an $85 \%$ remission rate in the VSL\#3-treated groups. ${ }^{63,65-67}$ The most significant limitation in all five studies was small sample sizes, with the largest being composed of 40 patients and the smallest consisting of 15 patients. Additional limitations include the short duration of the trials, with one being 3 months, another being 9 months, and the rest being 12 months. This warrants 
subsequent larger double-blinded RCTs with a much longer duration of follow-up.

\section{Future directions: fecal microbiota transplantation}

Fecal microbiota transplantation (FMT) is the infusion of a fecal suspension from a healthy individual into the gastrointestinal tract of another person in an attempt to treat an illness. The process has been described for decades, with the first reported case in humans performed by Eiseman and colleagues ${ }^{75}$ in 1958 for pseudomembranous colitis. FMT has primarily been used to treat $C$. difficile infection, with cure rates of up to $95 \%$ in published studies. ${ }^{76}$ This novel technique began to be employed because of a continual rise in $C$. difficile infections since the year 2000, especially in patients with recurrent infections that failed standard antibiotics therapy, in theory because of bowel flora deficiencies. Given the reduced diversity of luminal microbiota in IBD and specific deficiencies in Bacteroidetes and Firmicutes in IBD patients, FMT offers an attractive solution to replenish those species. ${ }^{9}$ While probiotics may alter the metabolic or immunologic activity of residing native gut microbiota with a cultured single or a few strains, FMT seems to result in durable, long-term implantation of donor flora. ${ }^{77}$ Borody et al ${ }^{78}$ found marked success with FMT in six UC patients, causing reversal of inflammation in those cases. These cases required multiple infusions rather than the single episode of FMT that is typically done for $C$. difficile infection. A systematic review by Anderson et $\mathrm{al}^{79}$ in 2012 found no RCTs, but reviewed 17 case reports or case series for a total of 26 patients receiving FMT for management of their IBD. Of those, 27 patients had UC, 12 had CD, and two were unclassified. Unfortunately, there was variability and incomplete data for the preparation and administration of FMT. No article reported complete data enabling protocol replication. ${ }^{79}$ Variability was seen with the number of follow-up enemas - from 2 days to 59 weeks. There was also no standardization for reporting disease activity in most series. Twenty five patients had follow-up information and 19 of these reported complete resolution of their symptoms. Disease activity was reported only in 24 patients and 15 patients were in remission (15/24). Due to lack of standardized studies, clearly more rigorous studies are needed. More recently in 2013 Kunde et al ${ }^{80}$ performed a single-center uncontrolled study of FMT in 10 children with UC, showing safety, tolerability, and clinical response. They used a standardized Pediatric Ulcerative Colitis Activity Index ${ }^{81}$ to document clinical response and remission; the first study of its kind. Adverse events were similar to other studies and were reported as bloating, flatulence, abdominal pain, cramping, diarrhea, fatigue, fever, and bloody stools. One subject had disabling hematochezia in the third week after FMT, but it was thought to be due to a UC flare rather than temporally relating to the FMT.

Given the findings above, the evidence for use of FMT is limited and weak overall, with most data based on case reports. Although limited, this evidence shows promise that FMT may be an effective and safe alternative treatment for IBD patients. Certainly, FMT holds enormous potential for a wide range of diseases. Future directions should include fecal and mucosal microbial community profiling, as this would help determine whether FMT's mechanism of action in IBD is similar to that in $C$. difficile infection. Standardization of FMT preparation, donor selection, route of administration, and duration of therapy with FMT are all needed and should be established through double-blinded RCTs.

\section{Conclusions}

All of the Crohn's disease studies had significant limitations. The size of the study population in the majority of the studies was small. These studies were plagued by high dropout rates and lack of dose-response analyses. There was also lack of consistency with respect to concomitant use of other medications, including antidiarrheals, aminosalicylates, immunomodulators, and corticosteroids, which could have affected outcomes. None of these studies provide conclusive evidence of a beneficial role of probiotics in induction or maintenance of remission in CD. Larger RCTs are needed to show any benefit for the use of probiotics, prebiotics, or synbiotics for treatment of CD.

In the ulcerative colitis studies, various agents showed a trend toward improved rates in both induction of remission and maintenance in UC. The effect was noted both clinically and objectively on a macroscopic and molecular level, in conjunction with standard treatment. For pouchitis, the evidence appears to support a role for VSL\#3 as a maintenance therapeutic option when pouchitis is in remission. However, the limited data available do not support the use of probiotics as primary therapy for pouchitis.

\section{Disclosure}

The authors report no conflicts of interest in this work.

\section{References}

1. Kappelman MD, Rifas-Shiman SL, Kleinman K, et al. The prevalence and geographic distribution of Crohn's disease and ulcerative colitis in the United States. Clin Gastroenterol Hepatol. 2007;5(12):1424-1429. 
2. Burisch J, Jess T, Martinato M, Lakatos PL; ECCO-EpiCom. The burden of inflammatory bowel disease in Europe. J Crohns Colitis. 2013;7(4):322-337.

3. Sartor RB. Mechanisms of disease: pathogenesis of Crohn's disease and ulcerative colitis. Nat Clin Pract Gastroenterol Hepatol. 2006;3(7): $390-407$.

4. Sartor RB. Microbial influences in inflammatory bowel diseases. Gastroenterology. 2008;134(2):577-594.

5. Eckburg PB, Bik EM, Bernstein CN, et al. Diversity of the human intestinal microbial flora. Science. 2005;308(5728):1635-1638.

6. Zoetendal EG, von Wright A, Vilpponen-Salmela T, Ben-Amor K, Akkermans AD, de Vos WM. Mucosa-associated bacteria in the human gastrointestinal tract are uniformly distributed along the colon and differ from the community recovered from feces. Appl Environ Microbiol. 2002;68(7):3401-3407.

7. Sellon RK, Tonkonogy S, Schultz M, et al. Resident enteric bacteria are necessary for development of spontaneous colitis and immune system activation in interleukin-10-deficient mice. Infect Immun 1998;66(11):5224-5231.

8. Nagalingam NA, Lynch SV. Role of the microbiota in inflammatory bowel diseases. Inflamm Bowel Dis. 2012;18(5):968-984.

9. Frank DN, St Amand AL, Feldman RA, Boedeker EC, Harpaz N, Pace NR. Molecular-phylogenetic characterization of microbial community imbalances in human inflammatory bowel diseases. Proc Natl Acad Sci U S A. 2007;104(34):13780-13785.

10. Rioux KP, Madsen KL, Fedorak RN. The role of enteric microflora in inflammatory bowel disease: human and animal studies with probiotics and prebiotics. Gastroenterol Clin North Am. 2005;34(3) 465-482, ix.

11. Vanderpool C, Yan F, Polk DB. Mechanisms of probiotic action: implications for therapeutic applications in inflammatory bowel diseases. Inflamm Bowel Dis. 2008;14(11):1585-1596.

12. Damman CJ, Miller SI, Surawicz CM, Zisman TL. Microbiome and inflammatory bowel disease: is there a therapeutic role for fecal microbiota transplantation? Am J Gastroenterol. 2012;107(10): 1452-1459.

13. Meagher AP, Farouk R, Dozois RR, Kelly KA, Pemberton JH. J ileal pouch-anal anastomosis for chronic ulcerative colitis: complications and long-term outcome in 1310 patients. Br J Surg. 1998;85(6): 800-803.

14. Scaldaferri F, Gerardi V, Lopetuso LR, et al. Gut microbial flora, prebiotics, and probiotics in IBD: their current usage and utility. Biomed Res Int. 2013;2013:435268.

15. Veerappan GR, Betteridge J, Young PE. Probiotics for the treatment of inflammatory bowel disease. Curr Gastroenterol Rep. 2012;14(4):324-333.

16. Fedorak R, Demeria D. Probiotic bacteria in the prevention and the treatment of inflammatory bowel disease. Gastroenterol Clin North Am. 2012;41(4):821-842.

17. Jonkers D, Penders J, Masclee A, Pierik M. Probiotics in the management of inflammatory bowel disease: a systematic review of intervention studies in adult patients. Drugs. 2012;72(6):803-823.

18. Plein K, Hotz J. Therapeutic effects of Saccharomyces boulardii on mild residual symptoms in a stable phase of Crohn's disease with special respect to chronic diarrhea - a pilot study. Z Gastroenterol. 1993;31(2):129-134

19. Malchow HA. Crohn's disease and Escherichia coli. A new approach in therapy to maintain remission of colonic Crohn's disease? J Clin Gastroenterol. 1997;25(4):653-658.

20. Guslandi M, Mezzi G, Sorghi M, Testoni PA. Saccharomyces boulardii in maintenance treatment of Crohn's disease. Dig Dis Sci. 2000;45(7):1462-1464.

21. Prantera C, Scribano ML, Falasco G, Andreoli A, Luzi C. Ineffectiveness of probiotics in preventing recurrence after curative resection for Crohn's disease: a randomised controlled trial with Lactobacillus GG. Gut. 2002;51(3):405-409.

22. Schultz M, Timmer A, Herfarth HH, Sartor RB, Vanderhoof JA, Rath HC. Lactobacillus GG in inducing and maintaining remission of Crohn's disease. BMC Gastroenterol. 2004;4:5.
23. Bousvaros A, Guandalini S, Baldassano RN, et al. A randomized, double-blind trial of Lactobacillus GG versus placebo in addition to standard maintenance therapy for children with Crohn's disease. Inflamm Bowel Dis. 2005;11(9):833-839.

24. Marteau P, Lémann M, Seksik P, et al. Ineffectiveness of Lactobacillus johnsonii LA1 for prophylaxis of postoperative recurrence in Crohn's disease: a randomised, double blind, placebo controlled GETAID trial. Gut. 2006;55(6):842-847.

25. Van Gossum A, Dewit O, Louis E, et al. Multicenter randomizedcontrolled clinical trial of probiotics (Lactobacillus johnsonii, LA1) on early endoscopic recurrence of Crohn's disease after ileo-caecal resection. Inflamm Bowel Dis. 2007;13(2):135-142.

26. Chermesh I, Tamir A, Reshef R, et al. Failure of Synbiotic 2000 to prevent postoperative recurrence of Crohn's disease. Dig Dis Sci. 2007;52(2):385-389.

27. Garcia Vilela E, De Lourdes De Abreu Ferrari M, Oswaldo Da Gama Torres H, et al. Influence of Saccharomyces boulardii on the intestinal permeability of patients with Crohn's disease in remission. Scand J Gastroenterol. 2008;43(7):842-848.

28. Steed H, Macfarlane GT, Blackett KL, et al. Clinical trial: the microbiological and immunological effects of synbiotic consumption - a randomized double-blind placebo-controlled study in active Crohn's disease. Aliment Pharmacol Ther. 2010;32(7):872-883.

29. Benjamin JL, Hedin CR, Koutsoumpas A, et al. Randomised, doubleblind, placebo-controlled trial of fructo-oligosaccharides in active Crohn's disease. Gut. 2011;60(7):923-929.

30. Hafer A, Krämer S, Duncker S, Krüger M, Manns MP, Bischoff SC. Effect of oral lactulose on clinical and immunohistochemical parameters in patients with inflammatory bowel disease: a pilot study. $B M C$ Gastroenterol. 2007;7:36.

31. Bourreille A, Cadiot G, Le Dreau G, et al; FLORABEST Study Group. Saccharomyces boulardii does not prevent relapse of Crohn's disease. Clin Gastroenterol Hepatol. 2013;11(8):982-987.

32. Best WR, Becktel JM, Singleton JW, Kern F Jr. Development of a Crohn's disease activity index. National Cooperative Crohn's Disease Study. Gastroenterology. 1976;70(3):439-444.

33. Hyams JS, Ferry GD, Mandel FS, et al. Development and validation of a pediatric Crohn's disease activity index. J Pediatr Gastroenterol Nutr. 1991;12(4):439-447.

34. Goebell H. European cooperative Crohn's disease study (ECCDS): Evaluation of different activity indices and development of a new severity-activity index (SAI). Inflamm Bowel Dis. 1988;1988: 253-258.

35. Rutgeerts P, Geboes K, Vantrappen G, Beyls J, Kerremans R, Hiele M. Predictability of the postoperative course of Crohn's disease. Gastroenterology. 1990;99(4):956-963.

36. Tursi A, Brandimarte G, Giorgetti GM, Forti G, Modeo ME, Gigliobianco A. Low-dose balsalazide plus a high-potency probiotic preparation is more effective than balsalazide alone or mesalazine in the treatment of acute mild-to-moderate ulcerative colitis. Med Sci Monit. 2004;10(11):PI126-PI131

37. Kato K, Mizuno S, Umesaki Y, et al. Randomized placebo-controlled trial assessing the effect of bifidobacteria-fermented milk on active ulcerative colitis. Aliment Pharmacol Ther. 2004;20(10): 1133-1141.

38. Furrie E, Macfarlane S, Kennedy A, et al. Synbiotic therapy (Bifidobacterium longum/Synergy 1) initiates resolution of inflammation in patients with active ulcerative colitis: a randomised controlled pilot trial. Gut. 2005;54(2):242-249.

39. Sood A, Midha V, Makharia GK, et al. The probiotic preparation, VSL\#3 induces remission in patients with mild-to-moderately active ulcerative colitis. Clin Gastroenterol Hepatol. 2009;7(11):1202-1209, 1209.e1.

40. Tursi A, Brandimarte G, Papa A, et al. Treatment of relapsing mildto-moderate ulcerative colitis with the probiotic VSL\#3 as adjunctive to a standard pharmaceutical treatment: a double-blind, randomized, placebo-controlled study. Am J Gastroenterol. 2010;105(10) 2218-2227. 
41. Ng SC, Plamondon S, Kamm MA, et al. Immunosuppressive effects via human intestinal dendritic cells of probiotic bacteria and steroids in the treatment of acute ulcerative colitis. Inflamm Bowel Dis. 2010;16(8):1286-1298.

42. Matthes H, Krummenerl T, Giensch M, Wolff C, Schulze J. Clinical trial: probiotic treatment of acute distal ulcerative colitis with rectally administered Escherichia coli Nissle 1917 (EcN). BMC Complement Altern Med. 2010;10:13.

43. Li G, Zeng S, Liao W, Lv N. The effect of bifid triple viable on immune function of patients with ulcerative colitis. Gastroenterol Res Pract. 2012;2012:404752.

44. Chapman TM, Plosker GL, Figgitt DP. VSL\#3 probiotic mixture: a review of its use in chronic inflammatory bowel diseases. Drugs. 2006;66(10):1371-1387.

45. Rizzello F, Gionchetti P, Venturi A, Amadini C, Romagnoli R, Campieri M. Review article: monitoring activity in ulcerative colitis. Aliment Pharmacol Ther. 2002;16(Suppl)4:3-6.

46. Lichtiger S, Present DH, Kornbluth A, et al. Cyclosporine in severe ulcerative colitis refractory to steroid therapy. $N$ Engl $J$ Med. 1994;330(26):1841-1845.

47. Harig JM, Soergel KH, Komorowski RA, Wood CM. Treatment of diversion colitis with short-chain-fatty acid irrigation. $N$ Engl J Med. 1989;320(1):23-28.

48. Schroeder KW, Tremaine WJ, Ilstrup DM. Coated oral 5-aminosalicylic acid therapy for mildly to moderately active ulcerative colitis. A randomized study. NEJM. 1987;317(26):1625-1629.

49. Kruis W, Schütz E, Fric P, Fixa B, Judmaier G, Stolte M. Double-blind comparison of an oral Escherichia coli preparation and mesalazine in maintaining remission of ulcerative colitis. Aliment Pharmacol Ther. 1997;11(5):853-858.

50. Rembacken BJ, Snelling AM, Hawkey PM, Chalmers DM, Axon AT. Non-pathogenic Escherichia coli versus mesalazine for the treatment of ulcerative colitis: a randomised trial. Lancet. 1999;354(9179): 635-639.

51. Ishikawa H, Akedo I, Umesaki Y, Tanaka R, Imaoka A, Otani $T$. Randomized controlled trial of the effect of bifidobacteria-fermented milk on ulcerative colitis. J Am Coll Nutr. 2003;22(1):56-63.

52. Kruis W, Fric P, Pokrotnieks J, et al. Maintaining remission of ulcerative colitis with the probiotic Escherichia coli Nissle 1917 is as effective as with standard mesalazine. Gut. 2004;53(11):1617-1623.

53. Cui HH, Chen CL, Wang JD, et al. Effects of probiotic on intestinal mucosa of patients with ulcerative colitis. World $J$ Gastroenterol. 2004;10(10):1521-1525.

54. Zocco MA, dal Verme LZ, Cremonini F, et al. Efficacy of Lactobacillus GG in maintaining remission of ulcerative colitis. Aliment Pharmacol Ther. 2006;23(11):1567-1574.

55. Fujimori S, Gudis K, Mitsui K, et al. A randomized controlled trial on the efficacy of synbiotic versus probiotic or prebiotic treatment to improve the quality of life in patients with ulcerative colitis. Nutrition. 2009;25(5):520-525.

56. Miele E, Pascarella F, Giannetti E, Quaglietta L, Baldassano RN, Staiano A. Effect of a probiotic preparation (VSL\#3) on induction and maintenance of remission in children with ulcerative colitis. Am J Gastroenterol. 2009;104(2):437-443.

57. Wildt S, Nordgaard I, Hansen U, Brockmann E, Rumessen JJ. A randomised double-blind placebo-controlled trial with Lactobacillus acidophilus La-5 and Bifidobacterium animalis subsp. lactis BB-12 for maintenance of remission in ulcerative colitis. $J$ Crohns Colitis. 2011;5(2):115-121.

58. Hegazy SK, El-Bedewy MM. Effect of probiotics on pro-inflammatory cytokines and NF-kappaB activation in ulcerative colitis. World $J$ Gastroenterol. 2010;16(33):4145-4151.

59. Ishikawa $\mathrm{H}$, Matsumoto $\mathrm{S}$, Ohashi $\mathrm{Y}$, et al. Beneficial effects of probiotic bifidobacterium and galacto-oligosaccharide in patients with ulcerative colitis: a randomized controlled study. Digestion. 2011;84(2): $128-133$.
60. Oliva S, Di Nardo G, Ferrari F, et al. Randomised clinical trial: the effectiveness of Lactobacillus reuteri ATCC 55730 rectal enema in children with active distal ulcerative colitis. Aliment Pharmacol Ther. 2012;35(3):327-334.

61. Guyatt G, Mitchell A, Irvine EJ, et al. A new measure of health status for clinical trials in inflammatory bowel disease. Gastroenterology. 1989;96(3):804-810.

62. Matts SG. The value of rectal biopsy in the diagnosis of ulcerative colitis. Q J Med. 1961;30:393-407.

63. Gionchetti P, Rizzello F, Venturi A, et al. Oral bacteriotherapy as maintenance treatment in patients with chronic pouchitis: a doubleblind, placebo-controlled trial. Gastroenterology. 2000;119(2): 305-309.

64. Kuisma J, Mentula S, Jarvinen H, Kahri A, Saxelin M, Farkkila M. Effect of Lactobacillus rhamnosus GG on ileal pouch inflammation and microbial flora. Aliment Pharmacol Ther. 2003;17(4):509-515.

65. Gionchetti P, Rizzello F, Helwig U, et al. Prophylaxis of pouchitis onset with probiotic therapy: a double-blind, placebo-controlled trial. Gastroenterology. 2003;124(5):1202-1209.

66. Mimura T, Rizzello F, Helwig U, et al. Once daily high dose probiotic therapy (VSL\#3) for maintaining remission in recurrent or refractory pouchitis. Gut. 2004;53(1):108-114.

67. Pronio A, Montesani C, Butteroni C, et al. Probiotic administration in patients with ileal pouch-anal anastomosis for ulcerative colitis is associated with expansion of mucosal regulatory cells. Inflamm Bowel Dis. 2008;14(5):662-668.

68. Calabrese C, Fabbri A, Gionchetti P, et al. Controlled study using wireless capsule endoscopy for the evaluation of the small intestine in chronic refractory pouchitis. Aliment Pharmacol Ther. 2007;25(11): $1311-1316$

69. Rahimi R, Nikfar S, Rahimi F, et al. A meta-analysis on the efficacy of probiotics for maintenance of remission and prevention of clinical and endoscopic relapse in Crohn's disease. Dig Dis Sci. 2008;53(9): 2524-2531.

70. Doherty GA, Bennett GC, Cheifetz AS, Moss AC. Meta-analysis: targeting the intestinal microbiota in prophylaxis for post-operative Crohn's disease. Aliment Pharmacol Ther. 2010;31(8):802-809.

71. Shen J, Zuo ZX, Mao AP. Effect of probiotics on inducing remission and maintaining therapy in ulcerative colitis, Crohn's disease, and pouchitis: meta-analysis of randomized controlled trials. Inflamm Bowel Dis. 2014;20(1):21-35.

72. Mallon P, McKay D, Kirk S, Gardiner K. Probiotics for induction of remission in ulcerative colitis. Cochrane Database Syst Rev. 2007;(4):CD005573.

73. Naidoo K, Gordon M, Fagbemi AO, Thomas AG, Akobeng AK. Probiotics for maintenance of remission in ulcerative colitis. Cochrane Database Syst Rev. 2011;(12):CD007443.

74. Elahi B, Nikfar S, Derakhshani S, Vafaie M, Abdollahi M. On the benefit of probiotics in the management of pouchitis in patients underwent ileal pouch anal anastomosis: a meta-analysis of controlled clinical trials. Dig Dis Sci. 2008;53(5):1278-1284.

75. Eiseman B, Silen W, Bascom GS, Kauvar AJ. Fecal enema as an adjunct in the treatment of pseudomembranous enterocolitis. Surgery. 1958;44(5):854-859.

76. Borody TJ, Campbell J. Fecal microbiota transplantation: techniques, applications, and issues. Gastroenterol Clin North Am. 2012;41(4): 781-803.

77. Grehan MJ, Borody TJ, Leis SM, Campbell J, Mitchell H, Wettstein A. Durable alteration of the colonic microbiota by the administration of donor fecal flora. J Clin Gastroenterol. 2010;44(8):551-561.

78. Borody TJ, Warren EF, Leis S, Surace R, Ashman O. Treatment of ulcerative colitis using fecal bacteriotherapy. J Clin Gastroenterol. 2003;37(1):42-47.

79. Anderson JL, Edney RJ, Whelan K. Systematic review: faecal microbiota transplantation in the management of inflammatory bowel disease. Aliment Pharmacol Ther. 2012;36(6):503-516. 
80. Kunde S, Pham A, Bonczyk S, et al. Safety, tolerability, and clinical response after fecal transplantation in children and young adults with ulcerative colitis. J Pediatr Gastroenterol Nutr. 2013;56(6):597-601.
81. Turner D, Otley AR, Mack D, et al. Development, validation, and evaluation of a pediatric ulcerative colitis activity index: a prospective multicenter study. Gastroenterology. 2007;133(2):423-432.

\section{Publish your work in this journal}

Clinical and Experimental Gastroenterology is an international, peerreviewed, open access journal, publishing all aspects of gastroenterology in the clinic and laboratory, including: Pathology, pathophysiology of gastrointestinal disease; Investigation and treatment of gastointes tinal disease; Pharmacology of drugs used in the alimentary tract;
Immunology/genetics/genomics related to gastrointestinal disease. This journal is indexed on CAS. The manuscript management system is completely online and includes a very quick and fair peer-review system. Visit http://www.dovepress.com/testimonials.php to read real quotes from published authors.

Submit your manuscript here: http://www.dovepress.com/clinical-and-experimental-gastroenterology-journal 